PROCEEDINGS OF THE

AMERICAN MATHEMATICAL SOCIETY

Volume 128, Number 11, Pages 3231-3240

S 0002-9939(00)05590-8

Article electronically published on March 2, 2000

\title{
BLOCH CONSTANTS FOR PLANAR HARMONIC MAPPINGS
}

\author{
HUAIHUI CHEN, P. M. GAUTHIER, AND W. HENGARTNER
}

(Communicated by Albert Baernstein II)

\begin{abstract}
We give a lower estimate for the Bloch constant for planar harmonic mappings which are quasiregular and for those which are open. The latter includes the classical Bloch theorem for holomorphic functions as a special case. Also, for bounded planar harmonic mappings, we obtain results similar to a theorem of Landau on bounded holomorphic functions.
\end{abstract}

Since confirmation of the Bieberbach Conjecture by Louis de Branges, the outstanding open problem in classical complex analysis is perhaps that of determining the precise value of the Bloch constant for holomorphic mappings of the unit disc. At this time the best lower estimate is in [3. In this note, we consider the analogous problem of estimating the Bloch constant for harmonic mappings.

A harmonic mapping is a complex-valued harmonic function defined on a domain in the complex plane. Harmonic mappings have interesting links with geometric function theory, minimal surfaces and locally quasiconformal mappings. For a survey of harmonic mappings in the plane, see [2].

For a continuously differentiable function $f(z)=u(z)+i v(z), z=x+i y$, we use the common notations for its formal derivatives:

$$
f_{z}=\frac{1}{2}\left(f_{x}-i f_{y}\right), \quad f_{\bar{z}}=\frac{1}{2}\left(f_{x}+i f_{y}\right) ;
$$

then $f$ is a harmonic mapping if and only if $f$ is twice continuously differentiable and

$$
\Delta f=4 f_{z \bar{z}}=0 .
$$

Let $f$ be a harmonic mapping of a domain $G$. $f$ is said to be univalent or locally univalent if $f$ is one-to-one or locally one-to-one on $G$. It is known 5 that a harmonic mapping is locally univalent if and only if its Jacobian $J_{f}$ does not vanish anywhere. Such a result does not hold in higher dimensions [7]. If $G$ is simply connected, then $f$ can be written as $f=\bar{g}+h$, where $g$ and $h$ are holomorphic on $G$. Since $f_{z}=h^{\prime}$ and $f_{\bar{z}}=\overline{g^{\prime}}$,

$$
J_{f}=u_{x} v_{y}-u_{y} v_{x}=\left|f_{z}\right|^{2}-\left|f_{\bar{z}}\right|^{2}=\left|h^{\prime}\right|^{2}-\left|g^{\prime}\right|^{2} .
$$

Received by the editors December 14, 1998.

2000 Mathematics Subject Classification. Primary 30C99; Secondary 30C62.

Key words and phrases. Bloch constant, harmonic mappings.

This research was supported in part by NSFC(China), NSERC(Canada) and FCAR(Québec).

(C)2000 American Mathematical Society 
For a continuously differentiable function $f$, denote

$$
\begin{aligned}
& \Lambda_{f}=\max _{0 \leq \theta \leq 2 \pi}\left|f_{z}+e^{-2 i \theta} f_{\bar{z}}\right|=\left|f_{z}\right|+\left|f_{\bar{z}}\right|, \\
& \lambda_{f}=\min _{0 \leq \theta \leq 2 \pi}\left|f_{z}+e^{-2 i \theta} f_{\bar{z}}\right|=|| f_{z}|-| f_{\bar{z}}|| .
\end{aligned}
$$

Note that $\left|J_{f}\right|=\Lambda_{f} \lambda_{f}$. A harmonic mapping $f$ is said to be $K$-quasiregular $(K \geq 1)$ on a domain $G$ if $\Lambda_{f} \leq K \lambda_{f}$, or equivalently $\Lambda_{f}^{2} \leq K\left|J_{f}\right|$ or $\lambda_{f}^{2} \geq\left|J_{f}\right| / K$, holds everywhere on $G$ (many authors also require $J_{f} \geq 0$ ).

We denote the unit $\operatorname{disc}\{z:|z|<1\}$ by $D$ and a disc with center at the origin and radius $r$ by $D_{r}$. A harmonic mapping $f=\bar{g}+h$ of the unit disc can be expanded in a series

$$
f\left(r e^{i \theta}\right)=\sum_{n=-\infty}^{\infty} c_{n} r^{|n|} e^{i n \theta}, 0 \leq r<1,
$$

where $g(z)=\sum_{n=1}^{\infty} \bar{c}_{-n} z^{n}, h(z)=\sum_{n=0}^{\infty} c_{n} z^{n}$. We call $c_{n}=c_{n}(f)$ the coefficients of $f$. The class of univalent sense preserving harmonic mappings on $D$ normalized by $c_{0}=0$ and $c_{1}=1$ will be denoted by $S_{H} . S_{H}^{0}$ will denote the subclass with $c_{-1}=0$. J. Clunie and T. Sheil-Small proved a distortion theorem and a Koebe theorem for $S_{H}$ and $S_{H}^{0}$ : If $f \in S_{H}$, then

$$
|f(z)| \geq \frac{1}{4}\left(1-\left|c_{-1}(f)\right|\right) \frac{|z|}{(1+|z|)^{2}}, \text { for } z \in D,
$$

and, in particular, $D_{R_{0}} \subset f(D)$ with

$$
R_{0}=\frac{1}{16}\left(1-\left|c_{-1}(f)\right|\right) .
$$

If $f \in S_{H}^{0}$, then $c_{-1}(f)=0$ and the factor $1-\left|c_{-1}(f)\right|$ in the above will be cancelled.

If the assumption of univalence is omitted, the distortion theorem and the Koebe theorem are no longer true even for holomorphic functions. However, replacing the univalence condition on the holomorphic function by that of boundedness, Landau proved a Koebe type theorem giving a lower estimate, depending on the bound of the function, for a schlicht disc contained in the image and centered at the origin. In Section 2 of this note, we prove two theorems for bounded harmonic mappings similar to the Landau theorem for bounded holomorphic functions.

On the other hand, for normalized holomorphic functions without any additional restriction, there is still the Bloch theorem which asserts the existence of a positive constant $b$ such that for any normalized holomorphic function $f$ in $D$ the image $f(D)$ contains a schlicht disc of radius $b$. By a schlicht disc, we mean a disc which is the univalent image of some region in $D$. The Bloch constant is defined as the "best" such constant, that is, the supremum of such $b$. It turns out that in the harmonic case, some extra assumption is required other than the normalization in order that a Bloch theorem hold. For $K$-quasiregular harmonic mappings (even in higher dimension), Bochner [1 had already proved the existence of a Bloch constant, but gave no estimate. In Section 3, we estimate this Bloch constant in the planar case. In Section 4, we give examples to show that for general harmonic mappings, and, in dimension bigger than two, even for univalent harmonic mappings, there is no Bloch theorem. However, we employ our Bloch theorem for quasiregular mappings to obtain a Bloch theorem for open planar harmonic mappings. This contains the classical Bloch theorem for general holomorphic functions. 
First, we consider bounded harmonic mappings of the unit disc. The following Schwarz lemma for harmonic mappings plays a key role in the proofs of our results.

Theorem 1. Let $f$ be a harmonic mapping of the unit disc $D$ such that $f(0)=0$ and $f(D) \subset D$. Then

$$
\begin{gathered}
\Lambda_{f}(0) \leq \frac{4}{\pi} \\
\Lambda_{f}(z) \leq \frac{8}{\pi\left(1-|z|^{2}\right)}, \text { for } z \in D, \\
|f(z)| \leq \frac{4}{\pi} \arctan |z| \leq \frac{4}{\pi}|z|, \text { for } z \in D .
\end{gathered}
$$

Proof. For $0 \leq \theta \leq 2 \pi$, let $u_{\theta}=\Re\left\{e^{i \theta} f\right\}$ and let $v_{\theta}$ be the harmonic conjugate of $u_{\theta}$ with $v_{\theta}(0)=0$. Then $F_{\theta}=u_{\theta}+i v_{\theta}$ is a holomorphic function such that $F_{\theta}(0)=0$ and $F_{\theta}(D)$ is contained in the strip $|\Re w|<1$. A conformal mapping $F$ of $D$ onto the strip with $F(0)=0$ is written by

$$
F(z)=\frac{2 i}{\pi} \log \frac{1+z}{1-z}
$$

By the subordination principle, we have $F_{\theta}\left(D_{r}\right) \subset F\left(D_{r}\right)$ for $r<1$. This shows that

$$
\Re\left\{e^{i \theta} f(z)\right\}=\Re\left\{F_{\theta}(z)\right\} \leq \frac{4}{\pi} \arctan |z|, \text { for } z \in D .
$$

(3) is proved now, since $\theta$ may be arbitrary. (1) is just a direct consequence of (3). For a fixed $z^{\prime} \in D$, applying (1) to the function $\left(f\left(\left(z+z^{\prime}\right) /\left(1+\bar{z}^{\prime} z\right)\right)-f\left(z^{\prime}\right)\right) / 2$, $z \in D$, we obtain (2) with $z=z^{\prime}$. The theorem is proved.

We remark that item (3) of the above theorem was shown (using the same argument) by E. Heinz [4].

Theorem 2. Let $f$ be a harmonic mapping of the unit disc $D$ such that $f(0)=0$, $J_{f}(0)=1$ and $|f(z)|<M$ for $z \in D$. Then, $f$ is univalent on a disc $D_{\rho_{0}}$ with

$$
\rho_{0}=\frac{\pi^{3}}{64 m M^{2}},
$$

and $f\left(D_{\rho_{0}}\right)$ contains a schlicht disc $D_{R_{0}}$ with

$$
R_{0}=\frac{\pi}{8 M} \rho_{0}=\frac{\pi^{4}}{512 m M^{3}},
$$

where $m \approx 6.85$ is the minimum of the function $\left(3-r^{2}\right) /\left(r\left(1-r^{2}\right)\right)$ for $0<r<1$.

Proof. The function $\left(3-r^{2}\right) /\left(r\left(1-r^{2}\right)\right), 0<r<1$, attains its minimum $m \approx 6.85$ at $r_{0} \approx 0.63$. For $0 \leq \theta \leq 2 \pi$, the function

$$
\phi_{\theta}(z)=f_{z}(z)-f_{z}(0)+\left(f_{\bar{z}}(z)-f_{\bar{z}}(0)\right) e^{-2 i \theta}, z \in D,
$$

is harmonic and satisfies

$$
\begin{gathered}
\phi_{\theta}(0)=0 \\
\left|\phi_{\theta}(z)\right| \leq \Lambda_{f}(z)+\Lambda_{f}(0) \leq \frac{4 M}{\pi}\left(1+\frac{2}{1-|z|^{2}}\right)=\frac{4 M\left(3-|z|^{2}\right)}{\pi\left(1-|z|^{2}\right)}
\end{gathered}
$$


for $z \in D$, since, by (1) and (2),

$$
\begin{gathered}
\Lambda_{f}(0) \leq \frac{4 M}{\pi}, \\
\Lambda_{f}(z) \leq \frac{8 M}{\pi\left(1-|z|^{2}\right)}, \text { for } z \in D .
\end{gathered}
$$

In particular,

$$
\left|\phi_{\theta}(z)\right| \leq m_{1}=\frac{4 M\left(3-r_{0}^{2}\right)}{\pi\left(1-r_{0}^{2}\right)}, \text { for } z \in D_{r_{0}} .
$$

Now, we apply (3) to the function $\phi_{\theta}\left(r_{0} z\right) / m_{1}$ and obtain

$$
\left|\phi_{\theta}(z)\right| \leq \frac{16 M|z|}{\pi^{2}} \cdot \frac{\left(3-r_{0}^{2}\right)}{r_{0}\left(1-r_{0}^{2}\right)}=\frac{16 m M|z|}{\pi^{2}}, \text { for } z \in D_{r_{0}} .
$$

Note that

$$
1=J_{f}(0) \leq \Lambda_{f}(0)^{2} \leq \frac{16 M^{2}}{\pi^{2}}
$$

and so

$$
\rho_{0} \leq \frac{\pi}{4 m}<r_{0}
$$

Thus

$$
\left|\phi_{\theta}(z)\right| \leq \frac{16 m M|z|}{\pi^{2}}, \text { for } z \in D_{\rho_{0}} .
$$

To prove the univalence, let $z_{1}, z_{2} \in D_{\rho_{0}}$ be two distinct points and $z_{2}-z_{1}=$ $\left|z_{2}-z_{1}\right| e^{i \theta}$. We have

$$
\begin{gathered}
\left|f\left(z_{2}\right)-f\left(z_{1}\right)\right|=\left|\int_{\left[z_{1}, z_{2}\right]} f_{z}(z) d z+f_{\bar{z}}(z) \overline{d z}\right| \\
\geq\left|\int_{\left[z_{1}, z_{2}\right]} f_{z}(0) d z+f_{\bar{z}}(0) \overline{d z}\right|-\left|\int_{\left[z_{1}, z_{2}\right]}\left(f_{z}(z)-f_{z}(0)\right) d z+\left(f_{\bar{z}}(z)-f_{\bar{z}}(0)\right) \overline{d z}\right| \\
\geq \int_{\left[z_{1}, z_{2}\right]}\left|f_{z}(0)+f_{\bar{z}}(0) e^{-2 i \theta}\right| d s-\int_{\left[z_{1}, z_{2}\right]}\left|\phi_{\theta}(z)\right| d s \\
\int_{\left[z_{1}, z_{2}\right]}\left|f_{z}(0)+f_{\bar{z}}(0) e^{-2 i \theta}\right| d s \geq \lambda_{f}(0)\left|z_{2}-z_{1}\right|=\frac{J_{f}(0)}{\Lambda_{f}(0)}\left|z_{2}-z_{1}\right| \geq \frac{\pi}{4 M}\left|z_{1}-z_{2}\right|, \\
\int_{\left[z_{1}, z_{2}\right]}\left|\phi_{\theta}(z)\right| d s<\frac{16 m M \rho_{0}}{\pi^{2}}\left|z_{2}-z_{1}\right|=\frac{\pi}{4 M}\left|z_{1}-z_{2}\right| .
\end{gathered}
$$

This shows $f\left(z_{1}\right) \neq f\left(z_{2}\right)$.

In the same way, for any $z^{\prime}=\rho_{0} e^{i \theta} \in \partial D_{\rho_{0}}$, we have

The theorem is proved.

$$
\begin{gathered}
\left|f\left(z^{\prime}\right)\right| \geq \int_{\left[0, z^{\prime}\right]}\left|f_{z}(0)+f_{\bar{z}}(0) e^{-2 i \theta}\right| d s-\int_{\left[0, z^{\prime}\right]}\left|\phi_{\theta}(z)\right| d s \\
\geq \lambda_{f}(0) \rho_{0}-\frac{16 m M}{\pi^{2}} \int_{0}^{\rho_{0}} r d r \\
\geq \frac{\pi}{4 M} \rho_{0}-\frac{8 m M \rho_{0}^{2}}{\pi^{2}}=\frac{\pi}{8 M} \rho_{0} .
\end{gathered}
$$

If we replace the normalization $J_{f}(0)=1$ in the above theorem by the stronger one that $f_{\bar{z}}(0)=0$ and $f_{z}(0)=1$, we may obtain a better conclusion. 
Theorem 3. Let $f$ be a harmonic mapping of the unit disc $D$ such that $f(0)=0$, $f_{\bar{z}}(0)=0, f_{z}(0)=1$, and $|f(z)|<M$ for $z \in D$. Then, $f$ is univalent on a disc $D_{\rho_{0}}$ with

$$
\rho_{0}=\frac{\pi^{2}}{16 m M},
$$

and $f\left(D_{\rho_{0}}\right)$ contains a schlicht disc $D_{R_{0}}$ with

$$
R_{0}=\rho_{0} / 2=\frac{\pi^{2}}{32 m M}
$$

where $m \approx 6.85$ is the number defined earlier.

Proof. The proof is almost the same as above. This time, we have

$$
\begin{gathered}
\int_{\left[z_{1}, z_{2}\right]}\left|f_{z}(0)+f_{\bar{z}}(0) e^{-2 i \theta}\right| d s \geq \lambda_{f}(0)\left|z_{2}-z_{1}\right|=\left|z_{1}-z_{2}\right|, \\
\int_{\left[z_{1}, z_{2}\right]}\left|\phi_{\theta}(z)\right| d s<\frac{16 m M \rho_{0}}{\pi^{2}}\left|z_{2}-z_{1}\right|=\left|z_{1}-z_{2}\right|,
\end{gathered}
$$

since the normalization implies $\lambda_{f}(0)=\Lambda_{f}(0)=1$. This shows the univalence. Also,

$$
\left|f\left(z^{\prime}\right)\right| \geq \lambda_{f}(0) \rho_{0}-\frac{16 m M}{\pi^{2}} \int_{0}^{\rho_{0}} r d r \rho_{0}-\frac{8 m M \rho_{0}^{2}}{\pi^{2}}=\frac{\rho_{0}}{2} .
$$

The theorem is proved.

Remark. The following example shows that the powers $M^{2}$ and $M^{3}$ in Theorem 2 are best possible. For $M>4$, define

$$
f(z)=\frac{4}{M} x-\frac{M}{4}\left(x^{2}-y^{2}\right)+\frac{M}{4} y i, \quad z=x+i y .
$$

Then, $f(0)=0, J_{f}(0)=1$, and $|f(z)|<M$ for $z \in D$. It is easy to see that $f$ is univalent for $x<8 / M^{2}$, but not univalent in any neighbourhood of a point $z$ with $x=8 / M^{2}$. So, the largest disc $D_{\rho_{0}}$, in which $f$ is univalent, has radius $\rho_{0} \leq 8 / M^{2}$ and the largest schlicht disc $D_{R_{0}}$ centered at the origin and covered by $f(D)$ has radius $R_{0} \leq 16 / M^{3}$.

Recall that the classical Koebe type theorem of Landau says: Let $f$ be a holomorphic function on the unit disc $D$ with $f(0)=0$ and $f^{\prime}(0)=1$. If $|f(z)|<M$ for $z \in D$, then $f$ is univalent on $D_{\rho_{0}}$ with

$$
\frac{1}{2 M}<\rho_{0}=\frac{1}{M+\sqrt{M^{2}-1}} \leq \frac{1}{M},
$$

and $f\left(D \rho_{0}\right)$ covers a disc $D_{R_{0}}$ with

$$
\frac{1}{4 M}<R_{0}=M\left(\frac{1}{M+\sqrt{M^{2}-1}}\right)^{2} \leq \frac{1}{M} .
$$

Moreover, this result is sharp. Comparing this result for holomorphic functions with Theorem 3, we see that the power of $M$ in the lower bounds of $\rho_{0}$ and $R_{0}$ is the same for both the holomorphic case and harmonic case, and only the constants differ. 
For $K$-quasiregular harmonic mappings, we have a theorem like the classical Bloch theorem for holomorphic functions. That is, we can guarantee the existence of a schlicht disc in the image, whose radius is bounded below by some constant which depends on $K$ only, but this schlicht disc may not be centered at the origin. To this end, we need the following theorem, in which our hypothesis can be considered as the assumption that the mapping is of bounded Fréchet derivative.

Theorem 4. Let $f$ be a harmonic mapping of the unit disc $D$ such that $f(0)=0$, $\lambda_{f}(0)=1$ and $\Lambda_{f}(z) \leq \Lambda$ for $z \in D$. Then, $f$ is univalent on a disc $D_{\rho_{0}}$ with

$$
\rho_{0}=\frac{\pi}{4(1+\Lambda)}
$$

and $f\left(D_{\rho_{0}}\right)$ contains a schlicht disc $D_{R_{0}}$ with

$$
R_{0}=\frac{1}{2} \rho_{0}=\frac{\pi}{8(1+\Lambda)} .
$$

Proof. Let

$$
F(z)=\psi^{-1}(f(z)), \quad \psi(z)=f_{z}(0) z+f_{\bar{z}}(0) \bar{z} .
$$

Then, $d F(0)$ is the identity mapping and $\psi$ is an expansion, so

$$
\begin{gathered}
\lambda_{F}(0)=\Lambda_{F}(0)=1, \\
\Lambda_{F}(z) \leq \Lambda_{f}(z) \leq \Lambda, \text { for } z \in D .
\end{gathered}
$$

As in the proofs of Theorems 2 and 3 , for $0 \leq \theta \leq \pi$, we introduce the harmonic mappings

$$
\phi_{\theta}(z)=F_{z}(z)-F_{z}(0)+\left(F_{\bar{z}}(z)-F_{\bar{z}}(0)\right) e^{-2 i \theta}, z \in D .
$$

We have $\phi_{\theta}(0)=0$ and

$$
\left|\phi_{\theta}(z)\right| \leq \Lambda_{F}(z)+\Lambda_{F}(0) \leq 1+\Lambda, \text { for } z \in D
$$

Thus, by Theorem 1,

$$
\left|\phi_{\theta}(z)\right| \leq \frac{4}{\pi}(1+\Lambda)|z|, \text { for } z \in D
$$

and the inequality is strict for $z \neq 0$.

To prove the univalence, let $z_{1}, z_{2}$ be distinct points of $D_{\rho_{0}}$ and $z_{2}-z_{1}=$ $\left|z_{2}-z_{1}\right| e^{i \theta}$. We have

$$
\begin{gathered}
\left|F\left(z_{2}\right)-F\left(z_{1}\right)\right|=\left|\int_{\left[z_{1}, z_{2}\right]} F_{z}(z) d z+F_{\bar{z}}(z) \overline{d z}\right| \\
\geq\left|\int_{\left[z_{1}, z_{2}\right]} F_{z}(0) d z+F_{\bar{z}}(0) \overline{d z}\right|-\left|\int_{\left[z_{1}, z_{2}\right]}\left(F_{z}(z)-F_{z}(0)\right) d z+\left(F_{\bar{z}}(z)-F_{\bar{z}}(0)\right) \overline{d z}\right| \\
=\int_{\left[z_{1}, z_{2}\right]}\left|F_{z}(0)+F_{\bar{z}}(0) e^{-2 i \theta}\right| d s-\int_{\left[z_{1}, z_{2}\right]}\left|\phi_{\theta}(z)\right| d s, \\
\int_{\left[z_{1}, z_{2}\right]}\left|F_{z}(0)+F_{\bar{z}}(0) e^{-2 i \theta}\right| d s \geq \lambda_{F}(0)\left|z_{2}-z_{1}\right|=\left|z_{2}-z_{1}\right|, \\
\int_{\left[z_{1}, z_{2}\right]}\left|\phi_{\theta}(z)\right| d s<\frac{4}{\pi}(1+\Lambda) \rho_{0}\left|z_{2}-z_{1}\right|=\left|z_{2}-z_{1}\right| .
\end{gathered}
$$

This shows $F\left(z_{1}\right) \neq F\left(z_{2}\right)$. 
In the same way, for any $z^{\prime}=\rho_{0} e^{i \theta} \in \partial D_{\rho_{0}}$, we have

$$
\begin{gathered}
\left|F\left(z^{\prime}\right)\right| \geq \int_{\left[0, z^{\prime}\right]}\left|F_{z}(0)+F_{\bar{z}}(0) e^{-2 i \theta}\right| d s-\int_{\left[0, z^{\prime}\right]}\left|\phi_{\theta}(z)\right| d s \\
>\lambda_{F}(0) \rho_{0}-\frac{4}{\pi}(1+\Lambda) \int_{0}^{\rho_{0}} r d r \\
=\rho_{0}-\frac{4}{\pi}(1+\Lambda) \frac{\rho_{0}^{2}}{2}=\frac{\rho_{0}}{2} .
\end{gathered}
$$

Consequently, $F$ is univalent on $D_{\rho_{0}}$ and $F\left(D_{\rho_{0}}\right)$ contains $D_{R_{0}}$. The conclusion of the theorem follows since $\psi$ is an affine transformation with $\lambda_{\psi}=1$.

Theorem 5. Let $f$ be a $K$-quasiregular harmonic mapping of the unit disc $D$ such that $\lambda_{f}(0)=1$. Then $f(D)$ contains a schlicht disc of radius at least

$$
R_{1}=\frac{\pi}{8 \sqrt{2}(1+2 K)} .
$$

Proof. Without loss of generality, we assume that $f$ is also harmonic on the boundary $\partial D$. Then there exists a point $z_{0} \in D$ such that

$$
\left(1-|z|^{2}\right) \lambda_{f}(z) \leq\left(1-\left|z_{0}\right|^{2}\right) \lambda_{f}\left(z_{0}\right)=A \geq 1, \text { for } z \in D .
$$

Let $\phi$ be a Möbius transformation of $D$ onto itself with $\phi(0)=z_{0}$. Define $F(\zeta)=$ $f(\phi(\zeta)) / A$, for $\zeta \in D$. Since

$$
\left(1-|\zeta|^{2}\right) \lambda_{F}(\zeta)=\left(1-|\phi(\zeta)|^{2}\right) \lambda_{f}(\phi(\zeta)) / A
$$

we have $\lambda_{F}(0)=1$, and

$$
\left(1-|\zeta|^{2}\right) \lambda_{F}(\zeta) \leq 1 \text { for } \zeta \in D
$$

Let $G(\omega)=\sqrt{2} F(\omega / \sqrt{2})$, for $\omega \in D$. Note that $G$ is also $K$-quasiregular. Thus, $\lambda_{G}(0)=\lambda_{F}(0)=1$, and

$$
\Lambda_{G}(\omega) \leq K \lambda_{G}(\omega)=K \lambda_{F}(\omega / \sqrt{2})<2 K, \text { for } \omega \in D .
$$

Now, by the preceding theorem, we see that $G$ is univalent on $D_{\rho_{0}}$ with $\rho_{0}=$ $\pi /(4(1+2 K))$, and $G\left(D_{\rho_{0}}\right)$ contains a disc of radius $\pi /(8(1+2 K))$. Consequently, $f(D)$ contains a schlicht disc of radius at least $R_{1}$. The theorem is proved.

If the function is normalized by $J_{f}(0)=1$, the radius of the schlicht disc will be a little smaller.

Theorem 6. Let $f$ be a $K$-quasiregular harmonic mapping of the unit disc $D$ such that $J_{f}(0)=1$. Then $f(D)$ contains a schlicht disc of radius at least

$$
R_{1}=\frac{\pi}{8 \sqrt{2} \sqrt{K}(1+2 K)}
$$

Proof. We have

$$
\lambda_{f}(0) \geq \frac{\sqrt{J_{f}(0)}}{\sqrt{K}}=\frac{1}{\sqrt{K}} .
$$

Applying the above theorem to the function $f / \lambda_{f}(0)$ gives the conclusion of the theorem. 
Let $x=\left(x_{1}, \cdots, x_{n}\right), u=\left(u_{1}, \cdots, u_{n}\right)$ and $u=f(x)$ be a harmonic mapping of a domain of $\mathbb{R}^{n}$ into $\mathbb{R}^{n}$. We denote by $f^{\prime}(x)$ the matrix $\left(\partial x_{j} / \partial x_{k}\right)_{j, k=1, \cdots, n}$. The following example shows that there is no Bloch theorem for general (even univalent) harmonic mappings, with the normalization that $f^{\prime}(0)$ is the identity, in dimension 3 , and hence in any dimension $\geq 3$.

Let $I=(-1,1)$ and $P_{3}=I \times I \times I$ be the cube in $\mathbb{R}^{3}$. For $k>4$, define a harmonic mapping $f_{k}$ by $u_{1}=x_{1}, u_{2}=x_{2}$ and $u_{3}=x_{3}+k x_{1} x_{2}$. Then, $f_{k}$ maps $\mathbb{R}^{3}$ onto itself injectively, and $f_{k}^{\prime}(0)$ is the identity. For $u=\left(u_{1}, u_{2}, u_{3}\right) \in f_{k}\left(P_{3}\right)$, denote by $r_{k}(u)$ the radius of the largest ball of dimension 3 centered at $u$ and contained in $f_{k}\left(P_{3}\right)$. Let $r_{k}=\sup \left\{r_{k}(u): u \in f_{k}\left(P_{3}\right)\right\}$. We claim that $r_{k} \leq 2 / \sqrt{k}$ for $k>4$. To prove this claim, for given $k>4$ and $u^{\prime}=\left(u_{1}^{\prime}, u_{2}^{\prime}, u_{3}^{\prime}\right) \in f_{k}\left(P_{3}\right)$, we distinguish two different cases: (1) $\left|u_{1}^{\prime}\right| \geq 1 / \sqrt{k}$ or $\left|u_{2}^{\prime}\right| \geq 1 / \sqrt{k}$; (2) $\left|u_{1}^{\prime}\right|,\left|u_{2}^{\prime}\right|<1 / \sqrt{k}$. First assume $\left|u_{1}^{\prime}\right| \geq 1 / \sqrt{k}$. Let $p=\left\{u \in \mathbb{R}^{3}: u_{1}=u_{1}^{\prime}\right\}$. Then, by the definition of $f_{k}$, we have

$$
p \cap f_{k}\left(P_{3}\right)=\left\{u \in \mathbb{R}^{3}: u_{1}=u_{1}^{\prime},-1<u_{2}<1,-1+k u_{1}^{\prime} u_{2}<u_{3}<1+k u_{1}^{\prime} u_{2}\right\} .
$$

Since $\left|u_{1}^{\prime}\right| \geq 1 / \sqrt{k},\left|k u_{1}^{\prime}\right| \geq \sqrt{k}$, we see that $r_{k}\left(u^{\prime}\right)<1 / \sqrt{k}$. It also holds for $\left|u_{2}^{\prime}\right| \geq 1 / \sqrt{k}$. Now, let $\left|u_{1}^{\prime}\right|,\left|u_{2}^{\prime}\right|<1 / \sqrt{k}$. Without loss of generality, assume that $0 \leq u_{1}^{\prime}<1 / \sqrt{k}$. If $u^{\prime \prime}=\left(1 / \sqrt{k}, u_{2}^{\prime}, u_{3}^{\prime}\right) \notin f_{k}\left(P_{3}\right)$, then $r_{k}\left(u^{\prime}\right) \leq 1 / \sqrt{k}$ since $\left|u^{\prime}-u^{\prime \prime}\right|=1 / \sqrt{k}-u_{1}^{\prime} \leq 1 / \sqrt{k}$. If $u^{\prime \prime} \in f_{k}\left(P_{3}\right)$, then $r_{k}\left(u^{\prime}\right)<2 / \sqrt{k}$, since $\left|u^{\prime}-u^{\prime \prime}\right| \leq 1 / \sqrt{k}$, and $r_{k}\left(u^{\prime \prime}\right)<1 / \sqrt{k}$ as just proved. This proves our claim.

Now, we discuss planar harmonic mappings. It is easy to give examples to show that neither the normalization $f_{z}(0)=1$ nor the normalization $J_{f}(0)=1$ yields a Bloch theorem for general univalent planar harmonic mappings. The following example shows that there is no Bloch theorem for harmonic mappings even with both of these normalizations, that is, $f_{z}(0)=1$ and $f_{\bar{z}}(0)=0$. Note that imposing both of these normalizations is equivalent to requiring that $d f(0)$ be the identity mapping. Let $w=u+i v$ and $z=x+i y$. For $k>1$, define $f_{k}(z)$ by

$$
u=x, \quad v=\frac{1}{k} e^{k y} \sin k x .
$$

It is easy to see that for any integer $m, f_{k}$ maps the strip $(m-1) \pi / k<x<m \pi / k$ into the strip $(m-1) \pi / k<u<m \pi / k$ injectively, but maps the straight line $x=m \pi / k$ onto the single point $w=m \pi / k$. If we let $F_{k}(z)=f_{k}(\pi /(2 k)+z)$, then $\left(F_{k}\right)_{z}(0)=1$ and $\left(F_{k}\right)_{\bar{z}}(0)=0$. It is obvious that $F_{k}(D)$ contains no disc with radius bigger than $\pi /(2 k)$.

A mapping of a domain $G \subset \mathbb{C}$ is said to be open if it maps any open subset of $G$ to an open set in $\mathbb{C}$. A mapping is said to be light if no continuum (connected closed set containing more than one point) is mapped to a single point. A non-constant holomorphic mapping is both open and light. The mappings $F_{k}$ are neither open nor light. Now we are going to prove a Bloch theorem for open planar harmonic mappings with the above normalization. First, we prove the following lemma.

Lemma 1. If a planar harmonic mapping is open, then it is light.

Proof. Let $f$ be an open harmonic mapping of a domain $G$. We may assume that $f_{z}$ is not equal to 0 identically. Otherwise, $\bar{f}$ is holomorphic and, because of the openness of $f, f$ is not a constant. Hence, $f$ is light. Let $a(z)=\overline{f_{\bar{z}}(z)} / f_{z}(z)$. Then $a$ is a meromorphic function on $G$ since both $\overline{f_{\bar{z}}}$ and $f_{z}$ are holomorphic. 
Suppose that $f$ is not light. Then, without loss of generality, we may assume that there is a continuum $E \subset G$ such that $f(z)=0$ for $z \in E$. We may choose a point $z_{0} \in E$ such that $f_{z}\left(z_{0}\right) \neq 0$. Then, $a\left(z_{0}\right)=e^{i \theta_{0}}$, since $J_{f}\left(z_{0}\right)=0$. $a$ is not equal to $e^{i \theta_{0}}$ identically. Otherwise, $f(G)$ will lie in a straight line making an angle $-\theta_{0} / 2$ with the positive real axis, which contradicts the openness. Replacing $z_{0}$ by another point very near it, if necessary, we may assume that $a^{\prime}\left(z_{0}\right) \neq 0$.

There exists a conformal mapping $\omega$ of the unit disc $D$ onto a small neighborhood of $z_{0}$, such that $|a(z)|=1,>1$ or $<1$ (hence, $J_{f}(z)=0,<0$ or $>0$ ), according to whether $z \in \omega(I), z \in \omega\left(D^{+}\right)$or $z \in \omega\left(D^{-}\right)$, where $I=(-1,1)$, and $D^{+}$ and $D^{-}$denote the upper and lower half discs (excluding the real diameter $I$ ). Then, $E \cap \omega(D) \subset \omega(I)$ and, consequently, there is a subsegment $I^{\prime}$ of $I$ such that $\omega\left(I^{\prime}\right) \subset E$, since $J_{f} \neq 0$ on $\omega(D) \backslash \omega(I)$ and $E$ is a continuum. Without loss of generality, assume that $I^{\prime}=I$. Now, $F=u+i v=f \circ \omega$ is an open harmonic mapping of the unit disc $D$, and $F(z)=0$ for $z \in I$.

Consider the holomorphic function $\phi(z)=u+i u^{*}$, where $u^{*}$ is the harmonic conjugate of $u$. $\phi$ cannot be a constant because of the openness of $F$. So, there is a point $z^{\prime} \in I$ such that $\phi^{\prime}\left(z^{\prime}\right) \neq 0$ and a small disc $\Delta \subset D$ centered at $z^{\prime}$ such that $\phi$ is univalent on $\Delta$. Let $\Delta \backslash I=\Delta^{+} \cup \Delta^{-}$. Since $u=0$ on $I, \phi(I)$ lies in the imaginary axis, and $\phi$ is univalent on $\Delta$, we see that $\phi\left(\Delta^{+}\right) \subset L^{+}$and $\phi\left(\Delta^{-}\right) \subset L^{-}$, or, conversely, $\phi\left(\Delta^{+}\right) \subset L^{-}$and $\phi\left(\Delta^{-}\right) \subset L^{+}$, where $L^{+}$and $L^{-}$denote the right and left half planes respectively. This means that $u(z)>0$ for $z \in \Delta^{+}$and $u(z)<0$ for $z \in \Delta^{-}$, or, conversely, $u(z)<0$ for $z \in \Delta^{+}$and $u(z)>0$ for $z \in \Delta^{-}$. By using the same reasoning for the function $v$, we obtain a small disc $\Delta_{1} \subset \Delta$ centered at a point $z^{\prime \prime} \in I$ such that $v(z)>0$ for $z \in \Delta_{1}^{+}$and $v(z)<0$ for $z \in \Delta_{1}^{-}$, or, conversely, $v(z)<0$ for $z \in \Delta_{1}^{+}$and $v(z)>0$ for $z \in \Delta_{1}^{-}$, where $\Delta_{1}^{+}$and $\Delta_{1}^{-}$denote the upper and lower half discs of $\Delta_{1}$. Note that $\Delta_{1}^{+} \subset \Delta^{+}$and $\Delta_{1}^{-} \subset \Delta^{-}$. Now there are 4 different cases. However, in any case, we can conclude that $F\left(\Delta^{+}\right)$and $F\left(\Delta^{-}\right)$lie in two different quadrants respectively. Recall that $F(I)=\{0\}$. This shows $F$ is not open on $D$. We reach a contradiction, and the lemma is proved.

Before proving our Bloch theorem for open planar harmonic mappings, we need to introduce the notion of interior transformaton. A continuous mapping of a domain in the plane is called an interior transformaton in the sense of Stoillow, if $f$ is both open and light. The famous Stoilow theorem [6] says that an interior transformaton $f$ is topologically holomorphic, that is, $f$ can be written in the form $f=F \circ \omega$, where $F$ is a non-constant holomorphic mapping and $\omega$ is a homeomorphism.

Theorem 7. Let $f$ be an open harmonic mapping of the unit disc $D$ normalized by $f_{z}(0)=1$ and $f_{\bar{z}}(0)=0$. Then $f(D)$ contains a schlicht disc of radius at least

$$
R_{1}=\frac{\pi \sqrt{2}}{16}(7-4 \sqrt{3}) \approx 0.02 .
$$

Proof. According to the above lemma, $f$ is light and, hence, $f$ is an interior transformaton in the sense of Stoïlow. By the Stoïlow theorem, $f$ can be written in the form $f=F \circ \omega$, where $F$ is a non-constant holomorphic mapping $F$ and $\omega$ is a homeomorphism. Let $E$ be the set of zeros of $F^{\prime}$. Then $E^{\prime}=\omega^{-1}(E)$ is a closed discrete set in $D$ and $f$ is locally univalent in $D \backslash E^{\prime}$. As we mentioned in Section 1 , a harmonic mapping is locally univalent if and only if its Jacobian $J_{f}$ does not vanish anywhere. Now, because of the normalization and the continuity of $J_{f}, J_{f}$ 
is positive and, consequently, $\left|f_{z}\right|>\left|f_{\bar{z}}\right|$ on $D \backslash E^{\prime}$. Then $a(z)=\overline{f_{\bar{z}}(z)} / f_{z}(z)$ is a holomorpic function on $D \backslash E^{\prime}$, and $|a(z)|<1$ for $z \in D \backslash E^{\prime}$. Hence, the singularities $E^{\prime}$ are removable and the strict inequality persists on $D$ by the maximum principle. Since $a(0)=0$ and $|a(z)|<1$ for $z \in D$, by the classical Schwarz lemma, $|a(z)|<r$ for $z \in D_{r}, 0 \leq r<1$. So,

$$
\frac{\Lambda_{f}}{\lambda_{f}}=\frac{\left|f_{z}\right|+\left|f_{\bar{z}}\right|}{\left|f_{z}\right|-\left|f_{\bar{z}}\right|}=\frac{1+|a|}{1-|a|} \leq \frac{1+r}{1-r}=K_{r}
$$

holds everywhere on $D_{r} \backslash E^{\prime}$. Because of the continuity, $\Lambda_{f} \leq K_{r} \lambda_{f}$ holds everywhere on $D_{r}$. This shows $f$ is $K_{r}$-quasiregular on $D_{r}$.

For a fixed $r<1$, applying Theorem 5 to the function $F(\zeta)=f(r \zeta) / r, \zeta \in D$, we see that $f\left(D_{r}\right)$ contains a schlicht disc with radius at least

$$
\frac{\pi}{8 \sqrt{2}} \cdot \frac{r}{1+2(1+r) /(1-r)}
$$

which has maximum $R_{1}$ at $r=2 \sqrt{3}-3$. This proves the theorem.

Remark. The assumption of "openness" in our Bloch theorem can be weakened a little to "openness outside a discrete and closed (with respect to $D$ ) set $E$ ". The same proof works. We see from the above proof that if a harmonic mapping is open, then it is topologically holomorphic. So, we may say that in our Bloch theorem, we essentially put the assumption that the function is topologically holomorphic. We have also proved that, if a harmonic mapping is open, then it is light. On the other hand, there are light harmonic mappings which are not open. The function defined in the remark after Theorem 3 is such a function. The functions $F_{k}$ defined in this section, however, are not light. So, one may expect that lightness together with the same normalization also yields a Bloch theorem. This is an open question.

\section{REFERENCES}

[1] S. Bochner, Bloch's theorem for real variables, Bull. Amer. Math. Soc. 52 (1946), 715-719. MR 8,204a

[2] D. Bshouty and W. Hengartner, Univalent harmonic mappings in the plane, Ann. Univ. Mariae Curie-Sklodowska Sect. A 48 (1994), 12-42. MR 96m:30025

[3] H. Chen and P. M. Gauthier, On Bloch's constant, J. Anal. Math. 69 (1996), 275-291. MR 97j:30002

[4] E. Heinz, On one-to-one harmonic mappings, Pacific J. Math. 9 (1959), 101-105. MR 21:3683

[5] H. Lewy, On the non-vanishing of the Jacobian in certain one-to-one mappings, Bull. Amer. Math. Soc. 42 (1936), 689-692.

[6] S. Stoïlow, Principes topologiques de la théorie des fonctions analytiques, 1938. GauthierVillars.

[7] J. C. Wood, Lewy's theorem fails in higher dimensions, Math. Scand. 69 (1991), 166. MR 93a:58024

Department of Mathematics, Nanjing Normal University, Nanjing, Jiangsu 210097, People's Republic of China

E-mail address: hhchen@njnu.edu.cn

Département de mathématiques et de statistique, Université de Montréal, Montréal, QuÉBEC, H3C 3J7, CANADA

E-mail address: gauthier@dms.umontreal.ca

Département de mathématiques, Université Laval, Québec, G1K 7P4, Canada

E-mail address: walheng@mat.ulaval.ca 